\title{
Fluid structure interaction of a vibrating circular plate in a bounded fluid volume: simulation and experiment
}

\author{
J. Hengstler \& J. Dual \\ Department of Mechanical and Process Engineering, ETH Zurich, \\ Switzerland
}

\begin{abstract}
The eigenfrequencies and damping of a vibrating circular plate resting in a finite fluid domain are studied. A plate which is clamped at the center and free along the edge is excited by a coil and the vibrations are measured with a laser scanning vibrometer. A 3D model of the plate is built up in ANSYS, using the experimental data as verification. The focus of this work is to investigate the influence of boundaries like a wall or a free water surface on the eigenfrequency of the plate. Also the damping of the circular plate for different water levels is measured.

Keywords: fluid structure interaction, circular plate, vibration, added mass effect.
\end{abstract}

\section{Introduction}

There are some papers in the literature where the fluid structure interaction of circular plates are studied. Bauer [1] investigated a clamped circular plate which fully covers a liquid filled container theoretically and numerically. Later Amabili [2] made comparisons to the work of Bauer and studied also sloshing modes in the case where the fluid filled container is larger than the circular plate. Kwak and Han [3] studied the same problem and made some experiments to validate their results. Amabili et al. [4] and Kwak and Amabili [5] studied the eigenfrequencies of a fully submerged circular plate neglecting the influence of the near bottom of their container and the free water surface theoretically and experimentally. Jeong and Kim [6] investigated a fully submerged circular plate in a closed container completely filled with fluid theoretically and numerically. Due to the fixed size of the container the variation of the water depth below changes also the water 
depth above the plate. For this reason they did not study the water depth on each side independently. Esmailzadeh et al. [7] investigated a circular plate which is in contact with a fluid on one side. The fluid has either a free surface or is covered with a rigid plate. They computed the eigenfrequencies of the first three modes for different water depths. They did not validate their results with experiments.

In view of previous research and because only very limited results have been given for the damping of the vibrations a free edge circular plate fully submerged in water was studied where the depth of the water can independently be varied above and below the plate. Numerical and experimental results will be presented.

\section{Numerical simulation}

The numerical simulations were done with ANSYS 12.1. The 3D model is built using the 3D 20-node structural solid element (SOLID186) for the circular plate and the 3D acoustic fluid (FLUID30) for the surrounding water. The eight node element has four degrees of freedom per node, three for the translations and one for the pressure. But the translations are only active at the interface. The element can be used for submerged structure dynamics. The fluid and the plate are meshed together with coincident nodes. Only small amplitudes are considered, therefore the linear theory can be used.

\section{Experimental setup}

In order to verify the numerical results, experiments have been performed with a circular plate with free edges resting in a steel tank as sketched in figure 1. The plate is screwed to a rod of steel with a diameter of $30 \mathrm{~mm}$. Two different plates were used with a radius of $190 \mathrm{~mm}$ and $199.6 \mathrm{~mm}$, respectively. Both have a thickness of $2 \mathrm{~mm}$, whereas the tank has an inner radius of $200 \mathrm{~mm}$. The material of the plate is stainless steel. The plate is excited with a coil which is connected to a frequency generator and a magnet polarized in the coil direction which is glued to the plate. In this way a contactless excitation can be realised. The magnet has a mass of $7.5 \cdot 10^{-4} \mathrm{~kg}$. Therefore the mass ratio of the magnet to the mass of the plate is about $0.04 \%$. For this reason the magnet has not to be taken into account in the model.

Contactless measurements of the vibrations were carried out with a laser scanning vibrometer (LSV) since this is a technique well known to have a minimum influence on the vibrating plate. For this reason the tank has a transparent cover made of polymethylmethacrylate (PMMA). To assign the peaks in the measured amplitude spectrum to the specific eigenmodes a grid of four concentric circles with 32 points per circle was scanned to visualize the eigenmodes with the software of the LSV. 


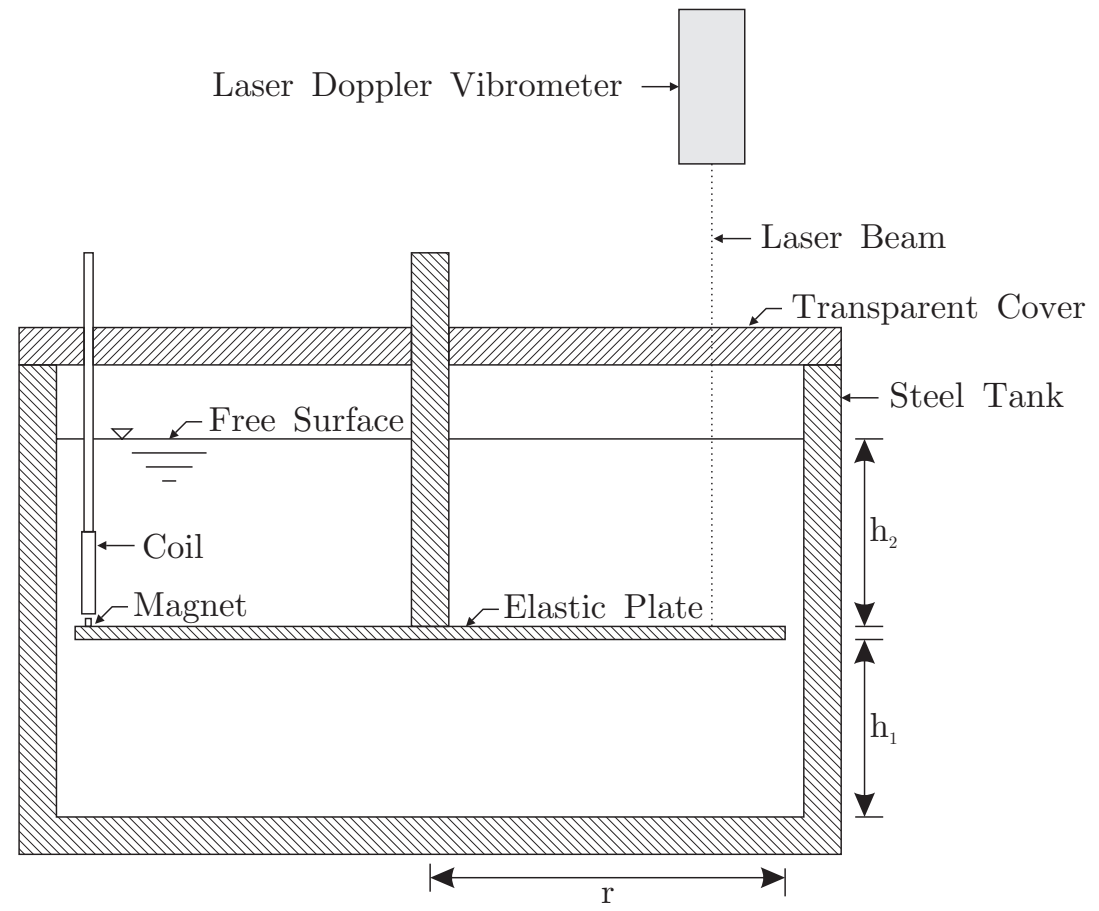

Figure 1: Experimental setup.

\section{Results}

\subsection{Eigenfrequency}

In order to measure the influence on a vibrating circular plate of a nearby wall or free water surface, experiments have been performed for a series of water depths $h_{1}$ and $h_{2}$ with a circular plate with a radius of $190 \mathrm{~mm}$. For the variation of $h_{1}$ the water depth $h_{2}$ was fixed at $100 \mathrm{~mm}$ and vice versa. The maximum ratio of the water depth to the wave length of the fluid is about $h_{1,2} / \lambda_{\text {Fluid }}=0.03 \ll$ 1. The modes of vibration will be named $(m-n)$ with $m$ being the number of nodal diameters and $\mathrm{n}$ the number of nodal circles, respectively. Because of the configuration used some modes have a very low amplitude leading to difficulties in identifying the eigenfrequency and modal damping. Because of limited space only a selection of modes are given. Especially the modes with nodal diameters have a high amplitude for the selected configuration which is typically about $10^{-4} \mathrm{~m} / \mathrm{s}$. In table 1 the measured and computed eigenfrequencies are listed for air and water with $h_{1,2}=100 \mathrm{~mm}$.

In figure 2 the results for varying depths are plotted. For the $\mathrm{x}$-axis the dimensionless ratio of $h_{1}$ and $h_{2}$ to the radius of the plate is selected. For the 

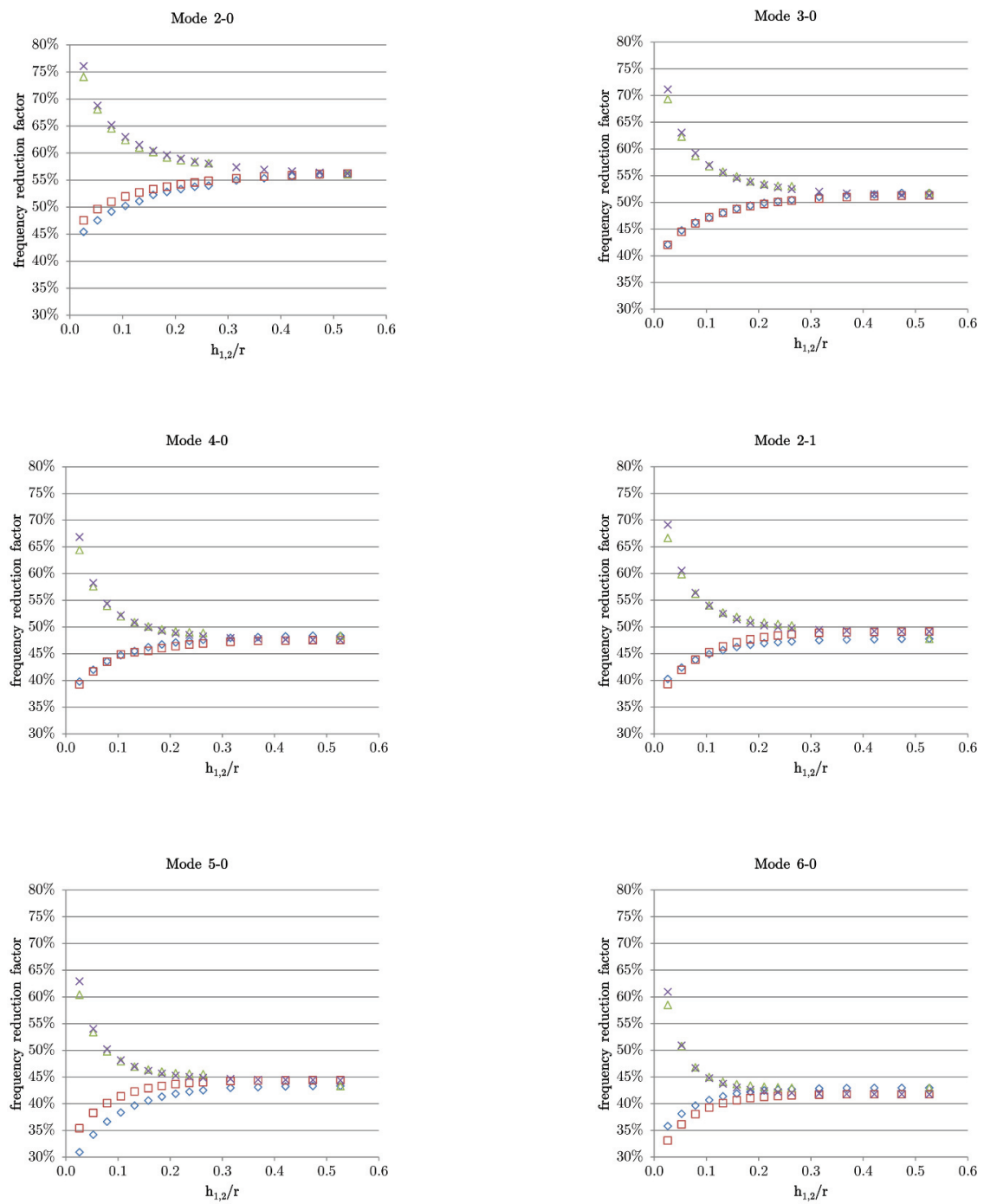

Figure 2: Experimental and numerical frequency reduction factor for a circular plate with free edges for different values of $h_{1,2} / r$ and modes m-n $\diamond$ - experimental variation of $h_{2}$ with $h_{1}=100 \mathrm{~mm}, \square$ - numerical variation of $h_{2}$ with $h_{1}=100 \mathrm{~mm}, \triangleleft$ - experimental variation of $h_{1}$ with $h_{2}=100 \mathrm{~mm}, \mathrm{X}$ - numerical variation of $h_{1}$ with $h_{2}=100 \mathrm{~mm}$. 
Table 1: Eigenfrequencies in $\mathrm{Hz}$ for a circular plate with free edges in air and in water for $h_{1,2} / r=0.53$ and different modes m-n.

\begin{tabular}{|r||r|r|r|r|}
\hline \multicolumn{1}{|c||}{} & \multicolumn{2}{c|}{ Air } & \multicolumn{2}{c|}{ Water } \\
\cline { 2 - 5 } & Experiment & Simulation & Experiment & Simulation \\
\hline \hline $2-0$ & 72.97 & 71.92 & 32.03 & 31.49 \\
\hline $3-0$ & 169.4 & 166.52 & 81.64 & 81.07 \\
\hline $4-0$ & 296.3 & 292.16 & 152.97 & 153.31 \\
\hline $2-1$ & 473.4 & 472.96 & 247.34 & 240.65 \\
\hline $5-0$ & 453.4 & 447.96 & 257.03 & 248.99 \\
\hline $6-0$ & 640.5 & 633.34 & 365.31 & 368.77 \\
\hline
\end{tabular}

$\mathrm{y}$-axis the dimensionless frequency reduction factor is used which is defined by

$$
\text { frequency reduction factor }=\frac{f_{\text {Res }}(\text { Air })-f_{\text {Res }}(\text { Water })}{f_{\text {Res }}(\text { Air })}
$$

As can be seen from figure 2 the eigenfrequencies of the investigated modes have an asymptotic behavior for the variation of $h_{1}$ as well as $h_{2}$. The asymptotic value is the one for an infinite fluid domain. For small $h_{2}$ the eigenfrequency is higher than the one in an infinite fluid domain which means it has a lower frequency reduction factor. For small $h_{1}$ the eigenfrequency is lower then the one in an infinite fluid domain which means a higher frequency reduction factor. For increasing nodal diameters $m$ the frequency reduction factor becomes smaller. The critical ratio $h_{1,2} / r$ where the influence of $h_{1}$ and $h_{2}$ on the eigenfrequency is negligible is also decreasing with increasing nodal diameters $m$ and has a value of about $h_{1,2} / r=0.56$ for the mode $2-0$ to $h_{1,2} / r=0.36$ for the mode $6-0$. For most cases the accuracy of the simulation is very good compared to the measurement. Especially the trend of the frequency reduction factor with varying $h_{1,2}$ fits very well.

Mode 2-1 which has an eigenfrequency in water near the one of mode 5-0 fits not directly in this series of the pure nodal diameter modes.

\subsection{Damping}

Damping is calculated from the phase and amplitude spectra of the transfer function between excitation and velocity signal for a circular plate with a radius of $199.6 \mathrm{~mm}$. For a higher accuracy of the measurement the harmonic response of the plate was measured at one point with a lock-in amplifier. The quality factor or $\mathrm{Q}$ factor is a dimensionless parameter which describes the damping. It is defined 
Table 2: Quality factors for a circular plate with free edges for different modes m-n and different media with $h_{1}=100 \mathrm{~mm}$ and different $h_{2}$.

\begin{tabular}{|r||r|r|r|r|}
\hline \multicolumn{1}{|c||}{} & \multirow{2}{*}{ Air } & \multicolumn{3}{|c|}{ Water $\left(\right.$ Height $\left.h_{2}\right)$} \\
\cline { 3 - 5 } & & $10 \mathrm{~mm}$ & $50 \mathrm{~mm}$ & $100 \mathrm{~mm}$ \\
\hline \hline $2-0$ & 890 & 34 & 25 & 17 \\
\hline $3-0$ & 1900 & 58 & 34 & 26 \\
\hline $4-0$ & 3190 & 92 & 52 & 33 \\
\hline $2-1$ & 1370 & 144 & 68 & 55 \\
\hline $5-0$ & 4260 & 110 & 70 & 60 \\
\hline $6-0$ & 7700 & 290 & 100 & 100 \\
\hline
\end{tabular}

by

$$
Q=\frac{f_{\text {Res }}}{f_{1}-f_{2}}
$$

where $f_{\text {Res }}$ is the eigenfrequency and $f_{1}$ and $f_{2}$ are the frequencies which correspond to a $45^{\circ}$ phase shift in the phase spectrum compared to the resonant phase and an amplitude shift of $\sqrt{2} / 2$ in the amplitude spectrum compared to the resonant amplitude, respectively, when a single degree of freedom system is adopted as a model. The damping ratio $\zeta$ is given by

$$
\zeta=\frac{1}{2 Q}
$$

The measurements in water have been done with $h_{1}$ fixed at $100 \mathrm{~mm}$ water below the circular plate and different water levels $h_{2}$ above. The values are listed in table 2.

According to table 2 the quality factor increases with an increasing number of nodal diameters $\mathrm{m}$. With an increasing water level above the quality factor decreases. From the three measurement points in water no conclusion can be made whether it is also an asymptotical behavior like in the case of the eigenfrequency. The total damping $Q_{\text {Total }}$ of the system which corresponds to the values in water from table 2 can be computed by

$$
\frac{1}{Q_{\text {Total }}}=\frac{1}{Q_{\text {Fluid }}}+\frac{1}{Q_{\text {Rest }}}
$$

where $Q_{\text {Fluid }}$ is the added damping from the surrounding fluid and $Q_{\text {Rest }}$ includes other damping effects like clamping, material damping etc. For increasing nodal diameters $m$ the value for $1 / Q_{\text {Fluid }}$ decreases, and the asymptotic value seems to be reached for larger $h_{2}$. 


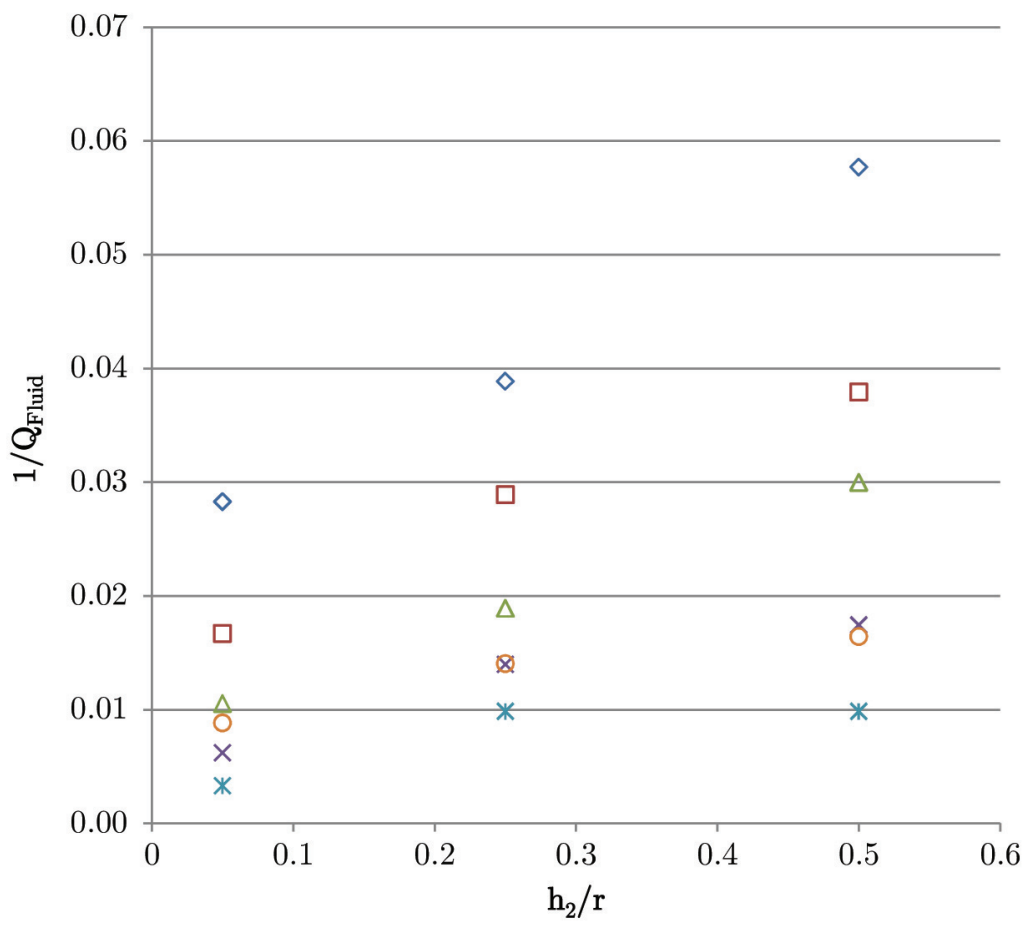

Figure 3: Experimental fluid damping for a circular plate with free edges for $h_{1} / r=0.5$ and different values of $h_{2} / r$ and modes m-n $\diamond$ - mode 2$0, \square$ - mode 3-0, $\triangleleft$ - mode $4-0, X$ - mode $2-1, O$ - mode 5 - 0 , $*$ - mode 6-0.

\section{Discussion}

While looking at figure 2 one obtains the impression that in an infinite fluid domain there is a subdomain around the vibrating plate which is in interaction with the plate and beyond this subdomain the fluid has no influence on the eigenfrequency of the vibrating plate any more. For the reduction of the eigenfrequency mainly the added mass effect is responsible. So if there is only little water around the plate this means that there is less added mass and the eigenfrequency increases. Unfortunately this explanation works only for the free water surface and not for a near wall. For the behavior when a wall is near the vibrating plate another explanation has to be found.

The higher quality factor with decreasing water level above can also be explained by the presence of less water in the relevant subdomain. 


\section{Conclusion}

It has been shown that numerical simulation with ANSYS with acoustic elements is a valid tool for computing the frequency reduction factor. It can also represent the effects of near walls and a free water surface. However the quality factor can not be computed with this model.

For a circular plate with free edges it was shown that boundaries which are more than $h / r \approx 0.5$ away can be neglected for the eigenfrequencies. The quality factor decreases with increasing water level above. It has to be further investigated if there is also an asymptotical behavior and a value of $h / r$ which can be defined for a limit of the relevant subdomain.

\section{Acknowledgements}

The authors acknowledge financial support from ETH Zurich and the Commission for Technology and Innovation CTI, Switzerland.

\section{References}

[1] Bauer, H., Coupled frequencies of a liquid in a circular cylindrical container with elastic liquid surface cover. Journal of Sound and Vibration, 180(5), pp. 689-704, 1995.

[2] Amabili, M., Vibration of circular plates resting on a sloshing liquid: solution of the fully coupled problem. Journal of Sound and Vibration, 245(2), pp. 261-283, 2001.

[3] Kwak, M. \& Han, S., Effect of fluid depth on the hydroelastic vibration of free-edge circular plate. Journal of Sound and Vibration, 230(1), pp. 171-185, 2000.

[4] Amabili, M., Dalpiaz, G. \& Santolini, C., Free-edge circular plates vibrating in water. Modal Analysis: the International Journal of Analytical and Experimental Modal Analysis, 10(3), pp. 187-202, 1995.

[5] Kwak, M. \& Amabili, M., Hydroelastic vibration of free-edge annular plates. Journal of Vibration and Acoustics, 121(1), pp. 26-32, 1999.

[6] Jeong, K. \& Kim, K., Hydroelastic vibration of a circular plate submerged in a bounded compressible fluid. Journal of Sound and Vibration, 283(1-2), pp. 153-172, 2005.

[7] Esmailzadeh, M., Lakis, A., Thomas, M. \& Marcouiller, L., Threedimensional modeling of curved structures containing and/or submerged in fluid. Finite Elements in Analysis and Design, 44(6-7), pp. 334-345, 2008. 